\title{
Antiaging strategy considering physiological characteristics
}

\author{
Hyoung-Woo Lee' ${ }^{1}$, Hyun-Seung Rhyu ${ }^{2 * *}$ \\ 'Department of Aero Mechanical Engineering, Jungwon University, Goesan, Korea \\ ${ }^{2}$ Department of Physical Education, Jungwon University, Goesan, Korea
}

Aging is a complex process that has a profound impact on health problems, and it is difficult to maintain a healthy daily life due to a gradual decrease in physiological function. In order to prevent aging, it is vital and valuable to study the reduction of physiological function. This study focused on the physiological factors associated with aging and discussed lifestyle changes to prevent aging. This study suggests if the national government should strive to develop and distribute programs, such as physical activity, cognitive function, dementia, and fall for the prevention of geriatric diseases, the old people will be able to live a healthier and happier life.

Keywords: Physiological characteristics, Physical activity, Cognitive function, Dementia, Aging

\section{INTRODUCTION}

The global population of elderly people is rapidly increasing, and also is the case in South Korea. Many studies are being conducted to clarify the mechanism of aging, but the causes of aging vary and therefore cannot be determined by a single theory. Aging is a complicated process that has a profound effect on health problems. Physiological functions of the body gradually decrease, making it difficult to maintain a healthy daily life (Ha and Son, 2018; Ha et al., 2018). To prevent aging, it is essential and worthwhile to study the reduction of the physiological function above all. Besides, it would be valuable to research prevention strategies of aging in consideration of these physiological characteristics. This study will review previous studies, focusing on the physiological factors related to aging and discuss lifestyle changes to prevent aging.

\section{PHYSIOLOGY FACTOR OF AGING}

As age increases, the physiological function decreases and the incidence of disease increases. The boundary between physiologi- cal changes due to aging and those directly or indirectly related to them is very unclear. Due to aging, the function of the human system is gradually deteriorating, and which is characterized by each physiological system.

\section{Musculoskeletal system}

Lean body mass peaks in their early 20 years for both males and females and remains at this level in the mid-forties. However, after the forties it tends to decline dramatically. Muscle weakness and muscle loss are significantly associated with aging and result in reductions in aerobic capacity, bone density, insulin sensitivity, metabolic rate, increased body fat, blood pressure cardiovascular disease, and increased risk of diabetes. Also, rest and reduction of exercise reduce the ability to exert strength and muscle contraction. Decreased muscle strength associated with aging in the lower body is associated with balance and mobility problems that lead to physical impairment and loss of independence. Muscle function is reduced by about $25 \%$ by 65 years and by about $40 \%$ during lifetime (Aoyagi and Shepard, 1992). Decreased myocardial function is due to a decrease of total muscle fiber, a reduction of
${ }^{*}$ Corresponding author: Hyun-Seung Rhyu (D) https://orcid.org/0000-0002-4987-7482 Department of Physical Education, Jungwon University, 85 Munmu-ro,

Goesan-eup, Goesan 28024, Korea

E-mail: rhyuhs@jwu.ac.kr

Received: April 23, 2019 / Accepted: May 29, 2019
This is an Open Access article distributed under the terms of the Creative Commons Attribution Non-Commercial License (http://creativecommons.org/licenses/by-nc/4.0/) which permits unrestricted non-commercial use, distribution, and reproduction in any medium, provided the original work is properly cited. 
muscle fiber size, an impairment of the stimulation-contraction dual system, and a reduction of high-threshold exercise muscle units. One of the critical consequences of a decrease in muscle mass and a decrease in the number of exercise units are muscle strength loss (Gleeson et al., 2003; Kirkendall and Garrett, 1998). Flexibility, which is the ability to move over the entire range of motion of the joints, is an important component of health associated with bones, muscles, and connective tissue. Some degenerative changes in the musculoskeletal system of joint movements are consequences of aging and long-term physical inactivity. In addition, the decreasing of lower leg strength and flexibility are closely related to postural fluctuations and are associated with a decline in gait function, such as a reduction in guarding and walking speed (Woollacott et al., 1986). Flexibility was reduced by $20 \%-50 \%$ between 30 and 70 years of age. Loss of flexibility also increases the likelihood of injury to the muscles that maintain joints and the risk of falls due to equilibrium and loss of stability increases (Barbosa et al., 2002). Bone density is seen through aging and loss of bone mass. Bone mineral density is stable until about 50 years of age, but after that gradually decreases calcium and bone regeneration ability. Especially during 5 years of menopause calcium begins to appear in women quickly (Eastell and Szulc, 2017).

\section{Cardiovascular system}

The aging process leads to the gradual degradation of the maximal oxygen uptake, which is caused by functional degeneration of almost all cardiovascular components, from the lungs to the skeletal muscle mitochondria (Hepple, 2000). The maximum aerobic capacity decreases with increasing age in both male and females regardless of physical fitness level. Between 25 and 65 years of age, the maximum oxygen uptake ability decreases by about $10 \%$ per decade and decreases over 80 years. Maximal cardiac output also decreases at about the same rate as the maximal oxygen uptake and could account for almost all of the maximal oxygen uptake associated with aging. At adulthood between 25 and 75 years of age, the maximum oxygen uptake was observed to decrease steadily by an average of $1 \%$ per year (Jackson et al., 1996). In addition, aging reduces the heart's autonomic nervous system (Perini et al., 2002) and increases blood pressure during resting and exercise. These parts are closely related to smoking, alcohol consumption, obesity, and an inactive lifestyle (Danaei et al., 2009).

\section{Respiratory system}

The efficiency of the lung decreases with age. The lung capacity is gradually reduced by 70 ; it is reduced by as much as $40 \%-$ $50 \%$. The ability of gas exchange inside lungs is continuously decreased with age. Between 30 and 70 years of age, maximum respiration is reduced by as much as $50 \%$, while residual volume is increased by 30\%-50\%. The lung capacity decreases by $40 \%-$ $50 \%$ (Carron et al., 2017). The major contributing factors are the progressive loss of elastic recoil within lung tissue. The chest wall becomes stiff, and there is a decrease in alveolar surface area. These changes diminish the efficiency of gas exchange and make it harder to exercise.

\section{Sensory nervous system}

The disorder of the nervous system is the most common cause of disability in over the 65 years elderly. The volume of the brain also decreases, the most critical areas of neurological change that occurs with aging are cognitive function, memory, movement speed, posture, balance, and gait (Yogev-Seligmann et al., 2008). Notably short-term memory power is decreased, and sensory motor control function such as visual and auditory function is also decreased. Although normal aging of the nervous system is accepted as inevitable. Which change in cognitive, motor control function, special senses are influenced by factors such as nutritional status, persistent intellectual stimulation and sensory stimulation. Moreover, neurological changes include loss of proprioception of the foot and ankle, loss of vibrational sensation of the foot, and loss of vestibular system function. Neurological changes include loss of proprioception of the foot and ankle, loss of vibrational sensation of the foot, and loss of vestibular system function. These changes continue to progressively increase with age and have a more significant impact on lower extremities than upper extremities. Depression also can be the result of impaired synaptic activity, which is also a common cause in the process of aging. Related studies indicate that about $25 \%$ of nursing home residents are clinically depressed.

\section{GUIDELINES FOR PREVENTION OF AGING}

Aging is an inevitable fate for humans. At this point in an aging society, more emphasis will be placed on how to live a healthy life and improve the quality of life than on how long it will last. Thus, preventing aging should focus on extending healthy lifespan rather than just life prolongation. The goal of prevention of aging is to maintain a healthy lifestyle as well as an improvement in lifestyle. The World Health Organization (WHO) defines health promotion as a process that enables people to improve their health 
by increasing their ability to manage their health and health determinants, rather than merely preventing and treating them. Besides, biological factors, environment, lifestyle, and medical system are considered to be the determinants of health through studies on the primary factors and causes of disease and death. Of these health determinants, lifestyle influence is about $52 \%$, and common lifestyle factors include physical activity (exercise), smoking, drinking, and nutrition. Above all, the importance of lifestyle in promoting health or health is that the determinants of the category of lifestyle can be controlled or managed by the individual's will, unlike biological factors (heredity), environment, and health care system. Therefore, a strategy to prevent aging should be a fundamental element of lifestyle, and it would be necessary to use appropriate medical services.

\section{No smoking}

According to the $\mathrm{WHO}$, million people die every year from direct or indirect smoking disease, and 10 million people are expected to die from tobacco-related illnesses by 2020 (World Health Organization, 2005). Health damage resulting from smoking includes lung cancer of $80 \%$ to $90 \%$, bladder cancer, myocardial infarction, $50 \%$ of brain cancer diseases, chronic bronchitis, emphysema, and chronic obstructive lung disease, all caused by smoking and $85 \%$ by smoking (Babizhayev and Yegorov, 2011). Although smoking rates are on the decline due to the smoking rates of all ages, the smoking rates of the younger generation are forecast to decline over the past decade, and the smoking rates of the younger age group are now as high as $12 \%$. Therefore, the more proactive antismoking policies should seek to reduce the smoking rates of all age groups, including the elderly, and the more appropriate awareness and awareness of the side effects of smoking among smokers is necessary (Cavelaars et al., 2000).

\section{Drinking habit}

Drinking alcohol in older adults is considered a risk factor in the physical and mentally healthy state in the United States. In the United States, the proportion of alcoholic beverages among the elderly population aged 65 and older is $33 \%$, of which $25 \%$ is estimated to drink alcohol every day (Hingson et al., 2005). In Korea, meanwhile, the percentage of people who drink alcohol was slightly higher than in the United States in 2009, while that of people over the age of 65 was somewhat higher than in the United States (Kim et al., 2013). Many people did not think of this as a problem for the elderly themselves or their neighbors. Older adults may experience faster increases in blood alcohol lev- els than younger people due to decreased age, metabolic function, plasma, body fluid levels. Lower alcohol intake can lead to drug and interaction due to chronic disease. Also, chronic alcohol abuse can cause not only physical problems such as falling, dementia, but also psychological issues such as family troubles, depression, and suicide, requiring active intervention to prevent and treat drinking problem (Ogden and Moskowitz, 2004). Therefore, although the need for abstinence may not be limited to old age, it can be considered necessary to have the correct type of abstinence because it can have a more significant negative effect.

\section{Improvement of eating habit}

Other than genetic factors, food, physical activity, stress response, rest and stability are all important variables in creating healthy aging. Eating right is an essential factor for healthy aging, as consuming food is a part of the same process that occurs every day and is necessary for survival. Generally, healthy aging requires the following eating habits. In old age, it is essential to reduce the amount of food first. Eating anything with a small amount of it is the most obvious way to reduce active oxygen and reduce inflammation. Of course, it is also important to eat while going through food types. However, if you do not reduce the amount, the effect of preventing active oxygen or inflammation decreases. We should eat foods that can reduce the number of calories and feel full. To do so, we need to increase the number of vegetarian dishes to the maximum. And foods that contain a lot of acidic substances should be reduced as much as possible. Foods with relatively high levels of acidic substances include soft drink, such as coke, cider, alcohol, and coffee, as well as animal-saturated fatty acids, kimchi, prepared food and sugar. On the other hand, there is a need to increase the intake rate of natural cholesterol-lowering diet. The soluble fiber in dried peas, peas, barley, oats, citrus fruits, and carrots acts as a cholesterol drop agent. And it is desirable to eat foods with lots of unsaturated fatty acids such as olive oil, canola oil, nuts, avocado, olives, almonds, peanuts, pecans, and macadamia than foods that contain saturated fatty acids. Unsaturated fatty acids are reported to play a role in reducing cholesterol in the body (Vijaimohan et al., 2006). Also, eating fresh vegetables and fruit, whole grains, nuts and seeds, fish and other foods with high-quality protein, green tea, and algae, as well as preventing the consumption of water at least $2 \mathrm{~L}$ per day, is crucial. In contrast, saturated fats, trans fats, refined sugar, flour, chemical seasoning, fast food, and alcohol are rather age-promoting foods, so it is necessary to limit consumption (Wu et al., 2014). 


\section{Participation in physical activity}

In general, aging involves the decline of physiological functions, including body structures, reduced body activity, and fat content, combined with decreased muscle and bone immune system, decreased muscle function, decreased digestive functions, decreased appetite, and lowered metabolic rates due to reduced muscle, respiratory and respiratory circulation during aging (Ahmed and Haboubi, 2010). It has been reported that proper physical activity or exercise can have the effect of preventing or delaying such side effects and preventing aging. For example, although aging can lead to rapid deterioration of the endocrine and immune systems, ongoing exercise can delay or prevent the aging process, along with increased endocrine and immune system functions. The positive effects of physical activity and exercise on the body are not only physiological effects, but also the emotional and psychological effects of depression, anxiety, and confidence on the performance of an elderly, and the sense of attachment to surrounding people (Fox, 1999).

\section{Stress management and regular health checkup}

Stress is a trigger for mental and physical illnesses due to negative emotions such as depression, which leads to inappropriate health behaviors such as increased chronic drinking and smoking, which adversely affects the health of individuals and organizations. In other words, excessive stress directly adversely affects the cardiovascular system, as well as secondary health risks through increased drinking and smoking. Therefore, it is crucial to look for proper measures to relieve stress. The following suggests nine creative solutions to stress. First, check the alternatives to resolve the stress one by one, then list the most realistic and desirable ones in order. Second, imagine the action to take. Third, implement the most feasible solution. Fourth, applaud yourself for your efforts. Fifth, solve the stress myself. Sixth, proceed with problem-solving in a concrete and viable way. Seventh, think broadly of all possible measures. Eighth, plan operations on what may happen in the future. Meanwhile, regular health checks are one of the most appropriate and proactive ways to prevent the deterioration of disease.

\section{CONCLUSIONS}

According to the National Statistical Office, Korea's population aged 65 and over is 657 million in 2015, 13.2\% of the total population is in aged society (more than 65\% of the population is over $14 \%$ ), and in 2026, (More than $20 \%$ of those aged 65 or old- er). Due to the increase in the elderly population, various social problems will also arise, especially the problems related to the elderly. The excessive medical expenditure due to illnesses such as various adult diseases and Alzheimer disease and the suicide due to the isolation and loneliness caused by a single family or an elderly couple will be serious social problems. Thus, the national government tries to develop an antiaging program considering the physiological characteristics of the elderly, and the people will become a real welfare state that lives wellness.

\section{CONFLICT OF INTEREST}

No potential conflict of interest relevant to this article was reported.

\section{REFERENCES}

Ahmed T, Haboubi N. Assessment and management of nutrition in older people and its importance to health. Clin Interv Aging 2010;5:207-216.

Aoyagi Y, Shephard RJ. Aging and muscle function. Sports Med 1992; 14:376-396.

Babizhayev MA, Yegorov YE. Smoking and health: association between telomere length and factors impacting on human disease, quality of life and life span in a large population-based cohort under the effect of smoking duration. Fundam Clin Pharmacol 2011;25:425-442.

Barbosa AR, Santarém JM, Filho WJ, Marucci Mde F. Effects of resistance training on the sit-and-reach test in elderly women. J Strength Cond Res 2002;16:14-18.

Carron M, Bertoncello F, Ieppariello G. Profile of sugammadex for reversal of neuromuscular blockade in the elderly: current perspectives. Clin Interv Aging 2017;13:13-24.

Cavelaars AE, Kunst AE, Geurts JJ, Crialesi R, Grötvedt L, Helmert U, Lahelma E, Lundberg O, Matheson J, Mielck A, Rasmussen NK, Regidor E, do Rosário-Giraldes M, Spuhler T, Mackenbach JP. Educational differences in smoking: international comparison. BMJ 2000;320:11021107.

Danaei G, Ding EL, Mozaffarian D, Taylor B, Rehm J, Murray CJ, Ezzati M. The preventable causes of death in the United States: comparative risk assessment of dietary, lifestyle, and metabolic risk factors. PLoS Med 2009;6:e1000058.

Eastell R, Szulc P. Use of bone turnover markers in postmenopausal osteoporosis. Lancet Diabetes Endocrinol 2017;5:908-923.

Fox KR. The influence of physical activity on mental well-being. Public Health Nutr 1999;2(3A):411-418.

Gleeson N, Eston R, Marginson V, McHugh M. Effects of prior concentric 
training on eccentric exercise induced muscle damage. Br J Sports Med 2003;37:119-125.

Ha MS, Kim JH, Kim YS, Kim DY. Effects of aquarobic exercise and burdock intake on serum blood lipids and vascular elasticity in Korean elderly women. Exp Gerontol 2018;101:63-68.

Ha MS, Son WM. Combined exercise is a modality for improving insulin resistance and aging-related hormone biomarkers in elderly Korean women. Exp Gerontol 2018;114:13-18.

Hepple RT. Skeletal muscle: microcirculatory adaptation to metabolic demand. Med Sci Sports Exerc 2000;32:117-123.

Hingson R, Heeren T, Winter M, Wechsler H. Magnitude of alcohol-related mortality and morbidity among U.S. college students ages 18-24: changes from 1998 to 2001. Annu Rev Public Health 2005;26:259-279.

Jackson AS, Wier LT, Ayers GW, Beard EF, Stuteville JE, Blair SN. Changes in aerobic power of women, ages 20-64 yr. Med Sci Sports Exerc 1996; 28:884-891.

Kim HT, Chun SS, Joung SH, Yun ME. Nutrient intake status of Korean drinkers: analysis of data from Korea National Health and Nutrition Examination Survey (KNHANES), 2011. J Korean Diet Assoc 2013;19: 343-355.

Kirkendall DT, Garrett WE Jr. The effects of aging and training on skeletal muscle. Am J Sports Med 1998;26:598-602.
Ogden EJ, Moskowitz H. Effects of alcohol and other drugs on driver performance. Traffic Inj Prev 2004;5:185-198.

Perini R, Fisher N, Veicsteinas A, Pendergast DR. Aerobic training and cardiovascular responses at rest and during exercise in older men and women. Med Sci Sports Exerc 2002;34:700-708.

Vijaimohan K, Jainu M, Sabitha KE, Subramaniyam S, Anandhan C, Shyamala Devi CS. Beneficial effects of alpha linolenic acid rich flaxseed oil on growth performance and hepatic cholesterol metabolism in high fat diet fed rats. Life Sci 2006;79:448-454.

Woollacott MH, Shumway-Cook A, Nashner LM. Aging and posture control: changes in sensory organization and muscular coordination. Int J Aging Hum Dev 1986;23:97-114.

World Health Organization. Preventing chronic diseases: a vital investment. WHO global report. Geneva (Switzerland): World Health Organization; 2005.

Wu G, Fanzo J, Miller DD, Pingali P, Post M, Steiner JL, Thalacker-Mercer AE. Production and supply of high-quality food protein for human consumption: sustainability, challenges, and innovations. Ann N Y Acad Sci 2014;1321:1-19.

Yogev-Seligmann G, Hausdorff JM, Giladi N. The role of executive function and attention in gait. Mov Disord 2008;23:329-342. 